\title{
A Survey on Green Scheduling Schemes for Homogeneous and Heterogeneous Cellular Networks
}

\author{
Ting Yang, Fabien Héliot, Chuan Heng Foh \\ Institute for Communication Systems, University of Surrey, \\ Guildford GU2 7XH, UK. Email: t.yang@surrey.ac.uk
}

\begin{abstract}
Energy-efficiency is becoming an important feature for designing the next generation of communication networks as are the multiplication of access points and the reduction of their coverage area. In this paper, we survey the latest development in energy-efficient scheduling, a.k.a. green scheduling, for both classic and heterogeneous cellular networks. We first introduce the main system model and framework that are considered in most of the existing green scheduling works. We then describe the main contributions on green scheduling as well as summarize their key findings. For instance, green scheduling schemes have demonstrated that they can significantly reduce the transmit power and improve the energy-efficiency of cellular systems. We also provide a performance analysis of some of the existing schemes in order to highlight some of the challenges that need to be tackled for making green scheduling more effective in heterogeneous networks. Indeed, the coordination between tiers and the rate fairness between the users of different tiers are important issues that have not been yet tackled. In addition, most of the existing design exhibits a computational complexity that is too high for being deployed in a real system.
\end{abstract}

Keywords-Green Scheduling, Coordination, Macro-only, Heterogeneous Network.

\section{INTRODUCTION}

$\mathbf{T}$ HE explosive growth of smart portable devices in recent years has triggered a shift from desktop to mobile internet access. It is envisioned that the next generation of mobile networks will support higher area capacity and will improve coverage. One effective solution to do so is to increase cell density for higher spatial reuse. This solution will inevitably introduce additional power consumption leading to a larger amount of greenhouse gas emission. With the information and communication technology (ICT) industry already contributing to $2 \%$ of world wide greenhouse emissions [1], seeking solutions to achieve energy efficiency (EE) in mobile networks has become a key consideration especially for the design of future mobile networks.

In a mobile network, base station (BS) accounts for as high as $80 \%$ of the total power consumption [2]. Typically, macrocell BSs use high transmit power to maintain its large cell size (500 m Inter Site Distance (ISD) for urban macrocell and $1732 \mathrm{~m}$ ISD for suburban macrocell in a Long Term Evolution (LTE) network [3]). While large cells suit low user density usage and help to reduce capital cost, they consume high energy to operate. Reducing cell size can help to lower energy consumption, but more cells are needed to cover the same area which, in turn, will increase capital cost and energy usage. The concept of heterogeneous network (HetNet) is proposed to offer a flexible solution. In HetNet, small cells are deployed within an existing macrocell and operate either concurrently with the macrocell by sharing the same radio resource or orthogonally, by using a different frequency band. Deploying small cells within areas of high traffic load can help to reduce traffic load of the macrocell. Intelligent network deployment strategies, where a high density deployment of low power small BSs is utilized, are believed to decrease the power consumption compared with a low density deployment of high power macro BSs. The idea being that a BS closer to mobile users requires less transmit power due to advantageous path loss conditions [4].

In a recent survey conducted by Feng et al. [4], it has been shown that EE transmissions offering lower power consumption are effective for saving significant amount of energy in a mobile network. Define EE to be the ratio of total rate to the total energy consumption of the network, the main objective of $\mathrm{EE}$ design is to maximize the value of $\mathrm{EE}$ measured in bit/Joule. The value of EE represents the amount of information bits that can be transmitted per every Joule of energy consumed by the network.

User scheduling and resource allocation have been found effective in the past for improving the spectrum efficiency (SE) or peak rate performance of communication system [2]. Given the increasing relevance of EE for future communication networks, EE-based scheduling and resource allocation have recently generated substantial research interests [2], [4], [5]. EE-based scheduling, which is also known as green scheduling (GS), aims at scheduling the user transmission while reducing the overall power consumption of the networks and guaranteeing an acceptable transmission rate. Traditionally, to achieve higher achievable rate, mobile networks operate at their maximum allowable power in order to achieve higher Signal to Interference and Noise Ratio (SINR). However, high transmit power does not necessarily lead to high network throughput; indeed, in a traditional multicell cellular system, higher transmit power will result in higher co-channel interference that can, counterproductively, reduce SINR. This highlights the importance of properly adjusting power according to the actual user requirements and environments, as it has recently been demonstrated in [2], [4], in order to maintain high transmission rate with lower transmit power. Whereas in a two-tier HetNet setup, given the different EE characteristics of small and the macrocells, the importance of fine-tuning the power according to user requirements/environment will be even more critical for 
controlling both inter and intra-tier interference. The HetNet setup provides additional flexibility in green scheduling to achieve further energy saving in transmissions, for instance, by performing joint scheduling between the two tiers for coordinating their interference and, in turn, improving EE. Some early investigations have already shown encouraging results of green scheduling in HetNets [6], [7].

However, we believe that the full potential of green scheduling for further improving $\mathrm{EE}$ in future communication networks, i.e. dense small cell networks, has yet to be unlocked. Thus, we provide here a detailed survey of green scheduling schemes for mobile networks and emerging HetNets by identifying and classifying the current trends in green scheduling and summarizing the key results of existing works. Based on the latter, we first identify the future challenges for green scheduling, and then propose our thoughts and ideas for tackling them. The rest of the paper is organized as follows. In Section II, we present the system model and framework that are common to existing green scheduling researches. Section III describes the various existing approaches in green scheduling. The key findings of these approaches are summarized and analysed, and then both single-tier and HetNet scenarios are simulated as an illustration of green scheduling schemes' performance in Section IV. The challenges and future directions in green scheduling design are discussed in Section $\mathrm{V}$, followed by important concluding remarks.

\section{SySTEM MODEL}

Green scheduling aims to achieve EE in addition to SE. In green scheduling, $\mathrm{EE}$ is often measured by the ratio of transmission rate to corresponding used power. Studies of green scheduling mostly consider a downlink Orthogonal Frequency Division Multiplexing Access (OFDMA) network with a single BS or $M$ coordinated BSs. Each BS accesses to the shared spectrum of $N$ subcarriers to serve $K$ users within its radio range. Both $\mathrm{BS}$ and user equipment (UE) are equipped with a single antenna. Availability of perfect channel state information (CSI) is often assumed in the process of green scheduling.

Energy efficiency of the system, EE, defined as the ratio of total transmission rate to the total consumed power, can be expressed as [4]

$$
\mathrm{EE} \triangleq \frac{R_{\text {total }}}{P_{\text {total }}}=\frac{\sum_{m=1}^{M} \sum_{k=1}^{K} R_{k(m)}}{\sum_{m=1}^{M} \sum_{n=1}^{N} \Delta_{m} p_{m}^{n}+P_{\text {fix }}},
$$

where $R_{k_{(m)}}$ represents the sum rate of user $k$ served by BS $m$. Green scheduling aims to maximize the number of transmitted bits with every joule of energy consumption. The power consumption consists of two parts, the transmit power $p_{m}^{n}$ and the operating power $P_{f i x}$, and $\Delta_{m}$ accounts for the Radio Frequency (RF) dependent slope of BS $m$. In (1), the quantity $P_{\text {fix }}$ captures the operating power consumption including circuit power, cooling system, power for backhaul communications, and others. The above formula is also valid for the small cell scenario with proper adjustments [4].

The objective of green scheduling is to find a particular user scheduling and power allocation such that the EE is maximized, given some constraints on power, rate, quality of service $(\mathrm{QoS})$, etc. It can be remarked that in most of the existing works on green scheduling for multicell systems [2], [5]-[10], the CSI, is assumed to be available at all BSs. This implicitly corresponds to a coordinated scenario, where BSs have enough backhaul capability to exchange this CSI. As for the single-cell systems, CSI is only available within each individual cell for its scheduling decision-making [11], [12].

\section{GREEN SCHEDULING SCHEMES}

The research on green scheduling generally exploits channel condition information to reduce transmit power while maintaining performance. Different techniques have been developed for different constraints and scenarios in the literature. We classify the existing research efforts by different constraints and then scenarios, as depicted in Fig. 1. The first track of research works generally imposes a constraint on transmit power when designing techniques for green scheduling [5], [7], [9]. To capture the minimum transmission rate requirement in the system, research works in the second track jointly consider power and rate constraints [2], [6], [8], [11], [12]. Apart from power and rate, the third track of research efforts focuses on fairness-aware green scheduling which aims to provide balanced rates among users [10], [13].

While majority of the existing works consider the traditional macrocell network as their scenario, some recent works have started to focus on proposing green scheduling schemes for the emerging HetNet scenario. In the case of multicell macro networks, BSs can perform coordinated green scheduling, i.e. BSs can coordinate their transmission to improve their EE. The scenario of HetNet is more complicated due to the involvement of two tiers. Ideally, interference among intra cells and between inter cells should be jointly considered when designing a green scheduling scheme, which can be interpreted as full coordination among two tiers. However, due to modeling complexity, many existing works in HetNet are only half-coordinated, i.e. developing green scheduling schemes for the small cells only while taking into account interference from the macro tier in their system model. For each track of research and scenario, we present several important works in the following.

\section{A. Power Constrained Green Scheduling}

For cellular networks sharing the same frequency, maximum transmit power for each BS is set for controlling interferences to other cells. This maximum transmit power is often derived during the planning phase with the objective to provide necessary coverage without excessive interference to other neighbouring cells. Traditionally, maximum transmit power is divided evenly on each subcarrier in OFDMA system. However, when channel condition is known, the corresponding transmit power can be calculated during the process of EE optimization, such that maximum transmit power may in effect not necessarily be used. The sum of scheduled power on each subcarrier must not exceed the maximum transmit power for each BS. Given power constraint and CSI knowledge, power constrained green scheduling assigns resources over time to maximize the EE of the system. 


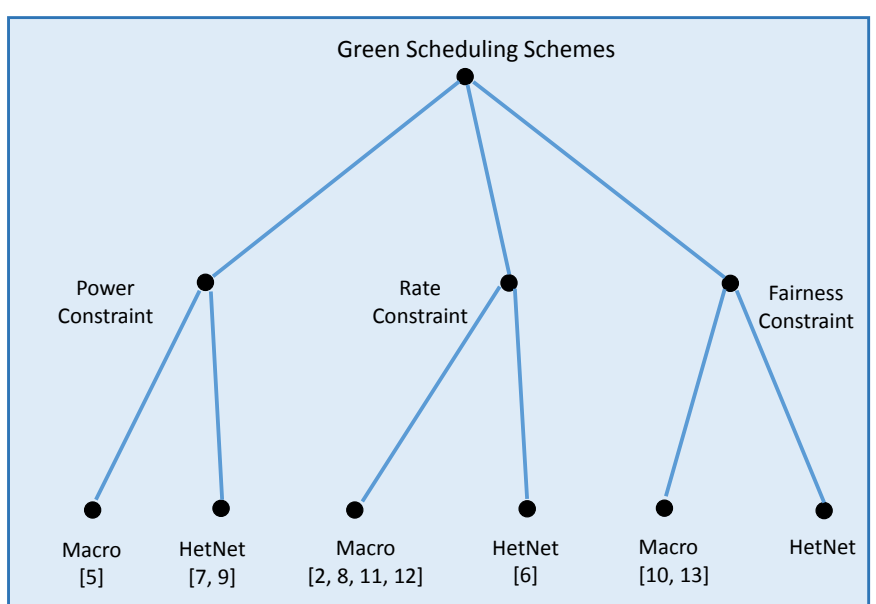

Fig. 1. Existing green scheduling schemes.

With the constraint on maximum transmit power, this track of research focuses on cooperative scheduling of users among neighboring BSs such that downlink transmit power can be reduced without significantly sacrificing data transmission rate. In [5], Venturino et al. consider both maximum overall BS transmission power as well as maximum transmit power per subcarrier. In their work, the power on each subcarrier should not exceed the overall BS transmission power divided by the number of subcarriers. Given the power constraint on each subcarrier, the corresponding EE on individual subcarriers is summed up to interchangeably represent the EE of the entire system. In other words, EE given by (1) is replaced by the following Sum-EE expression

$$
\text { Sum-EE } \triangleq \sum_{m} \sum_{n} \frac{R_{k(m)}^{n}}{\frac{\Delta_{m} p_{m}^{n}}{N}+\frac{P_{f i x}}{N}}
$$

The Sum-EE expression which adds up individual $\mathrm{EE}$ on subcarrier $n$ of BS $m$ is an approximation of the EE in (1), and hence it does not always maximize the EE. However, Sum-EE simplifies the introduction of weight on each subcarrier which permits the study of differentiated services [5].

In HetNet scenario, Zhang et al. consider maximizing EE in densely deployed femtocells with maximum transmit power per femtocell BS [7]. In their setup, macro and femto cells operate on different spectrum, and hence there is no interference between the two tiers. The research focuses on maximizing the $\mathrm{EE}$ of the femto tier. The technique used in the work is based on game theory. A distributed algorithm is developed to achieve the EE objective. In [9], Xiao et al. optimize the EE for HetNet by using Lagrangian dual decomposition, serial carrier and power allocation. The EE optimization is straightforwardly obtained from SE optimization with power constraints because fixed transmit/consumed power is considered in this work. The heterogeneity of BSs is used for further improving the EE of the network.

\section{B. Rate Constrained Green Scheduling}

Many power constraint EE optimization solutions favor users with good channel quality. Their solutions often allocate less resources to those users with poor channel quality than others which affects the QoS that the system attempts to deliver. A tradeoff between $\mathrm{EE}$ and $\mathrm{SE}$ exists [4]. In the literature, transmission rate is often the metric used to measure QoS. To deliver minimum rate in the system, i.e. to guarantee QoS, rate constraint is introduced to the EE optimization process. Given a scheduling period, the overall transmission of each BS must achieve a certain sum-rate and/or each user must be scheduled with a minimum transmission rate.

In the single-cell scenario, Xiao et al. in [11] focus on providing an optimal scheduling solution to maximize EE while satisfying the rate requirements requested by the users. The problem is first transformed from a fractional form into an equivalent subtractive form, then Lagrangian duality is used. An approximation of the problem is then used to obtain the optimal subcarrier allocation for the relaxed problem. In order to meet the rate constraint of all the users, the power is next allocated by using water-filling algorithm according to the obtained subcarrier allocation. For the same scenario, Zheng et al. in [12] study EE when maintaining a minimum sum-rate for the BS as well as minimum individual user rates. This green scheduling scheme employs water-filling method to find the best power allocation for each user. They propose a suboptimal algorithm for subcarrier allocation, which guarantees that each user is first allocated one subcarrier. Then, it assigns the rest of the subcarriers such that the power consumption is minimized. Their simulation results show that the algorithm can achieve a better balance between EE and SE with reduced complexity, compared with Xiong's algorithm in [13]. Whereas, in the multicell scenario, Heliot et al.'s scheme in [2] schedules a group of users that have similar CSI characteristics instead of individual users to achieve a low-complexity coordinated scheduling design. By comparing the EE performance of the non-coordinated and coordinated multicell approaches, they find that coordination helps to improve the EE of cellular systems.

Limited backhaul capacity has a fundamental impact on scheduling schemes, especially for small cells. In [8], Derrick et al. factor in the limitation of backhaul capacity in each macrocell BS and constrain the maximum overall sum-rate that each BS can transmit for a scheduling period. In their work, the optimization problem is transformed into the same form as in [11] for deriving an efficient iterative green scheduling algorithm for the multicell scenario.

In the HetNet scenario, interference caused by another tier can influence the design of green scheduling. In [6], Jiang et al. propose a green scheduling scheme to maximize the EE and satisfy the minimum rate requested by each user of the small cell in a single-cell HetNet, when taking into account the cross-tier interference from the macro-tier. The interference from the macro-tier may vary according to different CSI. In the meantime, an interference threshold is set for the smallcell tier in order to guarantee the QoS of the macrocell users. The optimal solution to the power and resource allocation 
is obtained by first transforming the EE problem into a subtractive form as in [8], [11], and then utilizing the dual decomposition method.

\section{Fairness Constrained Green Scheduling}

Introducing rate constraint in the EE optimization process has provided a basic means to deliver a certain service to BS or users. Since the minimum rate achievable by the system is not yet known during the EE optimization process, it is difficult to set a practical rate constraint for the optimization. Rate fairness that manages relative rates among users with predefined weights offers a practical setting in the EE optimization process.

In [13], Xiong et al. formulate EE optimization with predetermined weights to allow differentiated rate allocation among users. Their results show that with an appropriate setting of weights for users, fairness can be achieved, especially for users with low channel-gain-to-noise ratio (CNR). However, this work did not explicitly provide a method to appropriately set the weights that can achieve fairness. In order to support fairness, Ren et al. in [10] include proportional rate constraint in the EE optimization framework, and design a low complexity algorithm for solving it. The results show that system performances are better when users with higher CNR are scheduled more often, which in turn reflects that the proportional rate constraints influence the fairness between users. Both [10] and [13] demonstrate that without fairness consideration, the system would favor high CNR users.

\section{Performance of Green Scheduling Schemes}

In the following, we first summarize the key findings of our survey in Section III, before providing results demonstrating the potential of full coordination for green scheduling.

\section{A. Key Findings in Existing Green Scheduling Schemes}

Green scheduling schemes have shown to reduce the transmit power (e.g. by $90 \%$ in [2]) and improve the EE of the system (e.g. by $94.2 \%$ in [9]) compared with traditional scheduling [2], [9]-[13]. Optimal solutions to EE optimization have been developed for single cell scenario. However, these solutions are computationally complex [10], [12], [13]. Additional research efforts have been made to develop low complexity sub-optimal green scheduling with some achievements [10][13]. However, it is found that achieving EE targets introduces fairness unbalanced between different groups of users [11], [12]. In particular, users with poor channel condition transmit with unfairly low rates. Additional research efforts have been made to develop solutions for EE with QoS consideration.

For multicell networks where multiple cells share the same frequency band, it has been found that coordination among neighboring BSs can further improve EE performance [2]. However, due to inter-cell interference, finding optimal solutions requires computational complexity algorithms [5]. Suboptimal solutions can be obtained by using various approaches, such as relying on the symmetry of user locations [2] to reduce complexity.
Comparing with classic single network, HetNet has shown potential for significant EE improvement [9]. However, dedicated green scheduling schemes with cross-tier coordination have yet to be designed. Current green scheduling schemes considering HetNet are limited to a single tier coordination [7], [10].

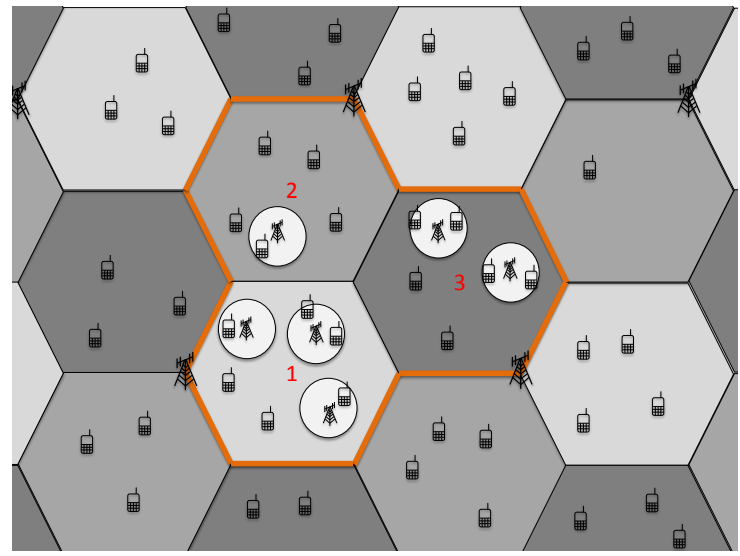

Fig. 2. Sectorized planar cellular system layout.

\section{B. Case Study of Green Scheduling Schemes}

Green scheduling techniques have shown to be effective for saving energy in a single tier mobile network, as reported in [2], [5], [8], [11], [13] for a classic cellular layout or in [6], [7] for a small-cell only layout; however, the effectiveness of these techniques in a two-tier HetNet scenario remains to be characterized. In order to examine the effectiveness of green scheduling in a two-tier HetNet scenario, and identify the limitations of the current existing schemes, we apply two of the various green scheduling schemes (i.e. "GS-Co" and "GS-NC") presented in this paper. The two green scheduling schemes are chosen such that the impact of cross-tier coordination can be observed. We also apply two traditional SEbased schedulers (i.e. "SE-Co" and "SE-Or") as benchmarks to see how EE optimization compares with traditional scheduling schemes. Further details about the four compared schedulers are given in Table I. We first consider a three-sector macroonly layout and then the two-tier HetNet layout as in [3], where sectorized macro BSs and uniformly distributed small BSs coexists in the orange dodecagonal area, as it is depicted in Fig. 2. Our results are obtained through Monte-Carlo simulations by using MATLAB. Moreover, we have considered the power/system parameters in Table $I$ of [2] and Tables 27 and 32 of [3] for plotting Figs. 3, 4 and 5, with $N=600$ subcarriers and $K=20$ uniformly distributed users in each sector of the dodecagonal area in Fig. 2. Regarding the power parameters for the small cells, we have used 0.13 Watt and 6.8 Watt for the maximum transmit power and circuit power, respectively and set the RF dependent slope to 4 .

In Figs. 3 and 4, we compare the EE performance of the four schemes with various ISD values for macro-only and HetNet with three randomly distributed small cells, respectively. 
TABLE I. GREEN SCHEDULING AND SE-BASED SCHEDULING SCHEMES

\begin{tabular}{|c|c|l|}
\hline Scheme & \multicolumn{1}{|c|}{ Property } & Description \\
\hline GS-Co & EE-based Coordinated & $\begin{array}{l}\text { Algorithm 5 of [5]. It has been specif- } \\
\text { ically designed for the classic cellular } \\
\text { layout; its generalized formulation made } \\
\text { it readily usable without modifications } \\
\text { for the two-tier scenario. }\end{array}$ \\
\hline GS-NC & EE-based Non-Coordinated & $\begin{array}{l}\text { The coordinated green scheduler in [2], } \\
\text { which has been specifically designed for } \\
\text { the classic cellular layout, is utilized } \\
\text { in two-tier HetNet scenario but without } \\
\text { cross tier coordination; in other words, } \\
\text { each tier is coordinated independently, } \\
\text { without being aware of cross-tier inter- } \\
\text { ference. }\end{array}$ \\
\hline SE-Co & SE-based Coordinated & $\begin{array}{l}\text { Algorithm 3 of [14], which is a coordi- } \\
\text { nated scheduler that can be readily used } \\
\text { for both macro and small cells. }\end{array}$ \\
\hline SE-Or & SE-based Non-Coordinated & $\begin{array}{l}\text { Each BS has an equal number of dedi- } \\
\text { cated subcarriers that are orthogonal to } \\
\text { each other. }\end{array}$ \\
\hline
\end{tabular}

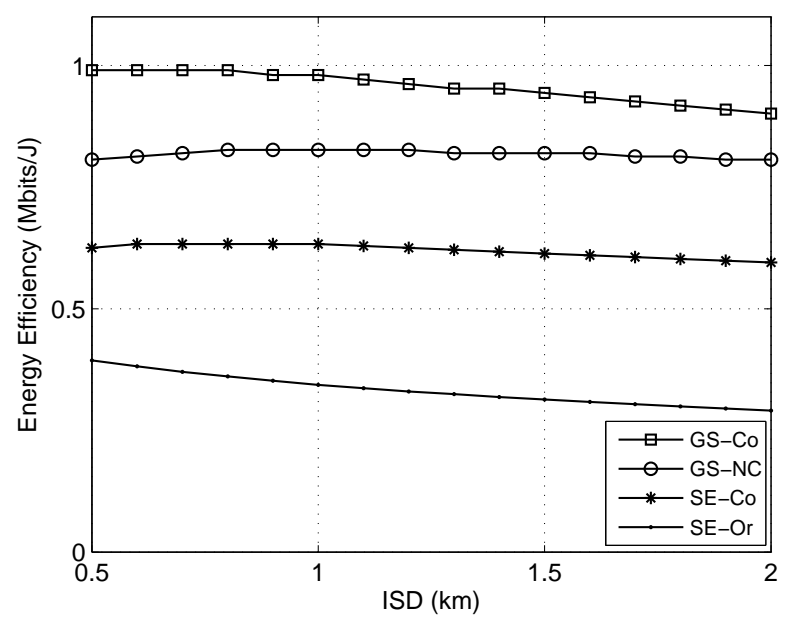

Fig. 3. EE performance comparison of various scheduling methods vs. the inter-site distance in one-tier macro-only layout.

For the macro-only scenario in Fig. 3, EE-based schedulers outperform SE-based schedulers. The "SE-Or" scheduler uses full power for transmission, though there is no interference between different sectors, the available frequency bands for each sector reduced by a third compared with other schedulers. It therefore has the worst EE performance [2]. The "SE-Co" scheduler can take advantage of available CSI to adjust its transmit power rather than transmitting at full power. However, since SE-based schedulers are not designed to improve EE, their performance are worse than "GS-NC" and "GS-Co" schedulers in terms of EE. The "GS-Co" scheduler has the best EE performance given that it uses CSI knowledge to optimize $\mathrm{EE}$ by enforcing BSs coordination.

Figure 4 shows the EE performance of the four schemes and the fairness rate distribution between tiers in HetNet, in the upper and lower parts of Fig. 4, respectively. We observe that "GS-Co" scheduler has the best EE performance. Interestingly, it can be remarked that SE coordination outperforms EE non-
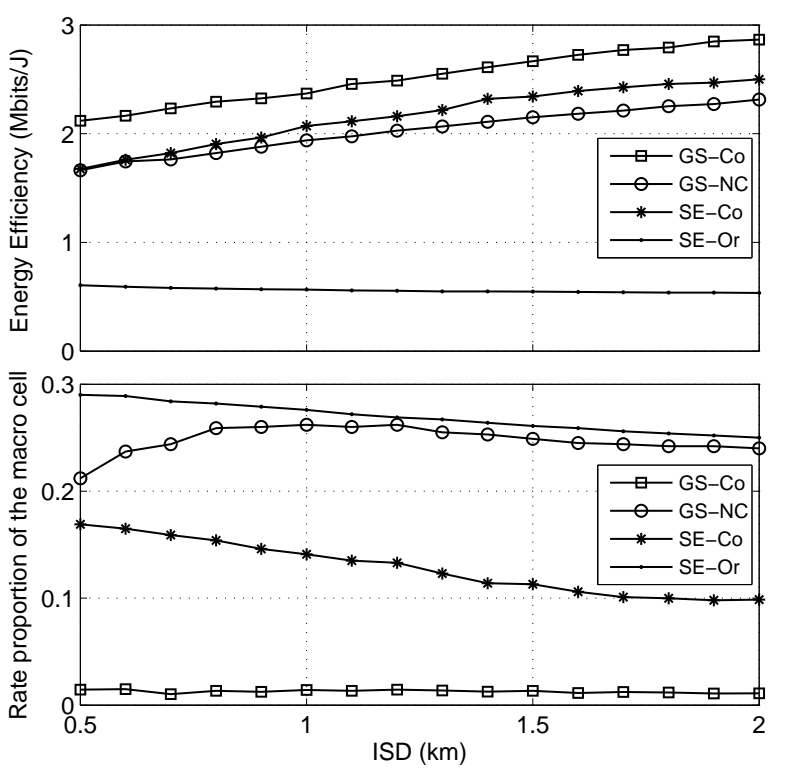

Fig. 4. Performance comparison of various scheduling methods in terms of $\mathrm{EE}$ and rate fairness vs. the inter-site distance in two-tier HetNet layout.

coordination in the HetNet scenario. The macro tier is not aware of the small cells for "GS-NC" scheme, the transmit power of the macro BSs will increase with the ISD, creating more interference to the small cells. In turn, small cells will increase their transmit power in order to combat the interference. Such behaviors consume more power and result in rate degradation due to both increased intra-tier and intertier interferences. On the contrary, "SE-Co" scheduler jointly considers the transit powers for both tiers such that interference can be minimized. This observation illustrates the importance of coordination among different tiers for improving EE. Our result confirms the benefit of coordination and sharing CSI between BSs, as it has been discussed in [15].

When comparing the results for the macro and HetNet scenarios in Figs. 3 and 4, it can be remarked that green schedulers provide an EE improvement of at least $100 \%$ in the HetNet scenario when compared to the macro-only scenario. This is because small cells in HetNet use lower transmission power to communicate with UEs while achieving similar rate than users served by the macrocells. This can be observed in (1) where adding similar rate elements in the numerator while reducing the level of the power in the denominator contributes to better EE performance. In addition, as it can be seen in Fig. 5, adding small cells have the effect of lowering the transmission power of coordinated schemes, and hence this reduces the interference, which in turn improves the rate and EE.

The lower part of Fig. 4 compares the rate proportion of the macrocell for the four schemes, which is an indicator of fairness on how fair is the rate distribution between the two tiers. It can be observed that some of the existing macro-only 
green scheduling solutions [2], [5], when directly applied to the HetNet scenario, generate unfair load distribution between the two tiers; this is mainly due to the different power and propagation characteristics of BSs in different tiers. Indeed, the results presented in the lower part of Fig. 4 indicate that "GSCo" achieves the best EE performance of the system by overfavoring the small cells at the expense of the macrocell rate. The same unfair load balancing problem can also be noticed in SE coordination although the impact is not as severe. On the other hand, both "GS-NC" and "SE-Or" can maintain a fairer load distribution between tiers as they do not perform cross-tier coordination. In summary, this result clearly shows the existence of a tradeoff between EE and load balancing in a multi-tier system. Even though, some existing macro-only green scheduler can be used to improve the EE in HetNet, they still suffer from unfair rate allocation problem. This indicates that green scheduling with cross-tier coordination requires additional attention. Even more so, when mentioning that the existing green coordinated scheduling schemes do not take into account the on/off switching capabilities of small cells; this certainly represents an extra degree of freedom for improving the EE but, as well, an extra challenge to make it work effectively.
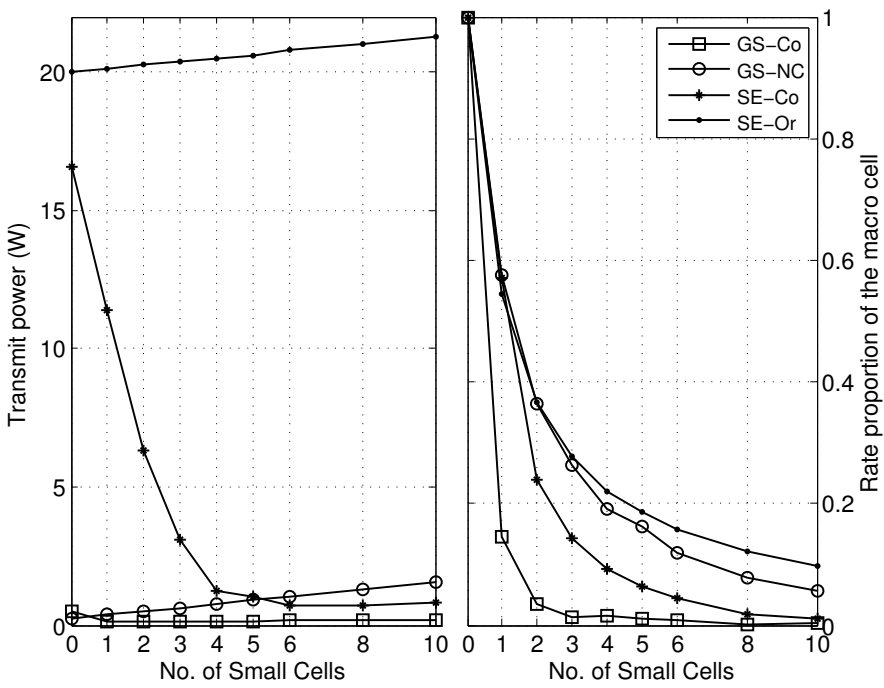

Fig. 5. Performance comparison of various scheduling methods in terms of transmit power and rate fairness vs. number of small cells.

Figure 5 illustrates the corresponding transmit power of the various schedulers as a function of the number of small cells within the dodecagonal area illustrated in Fig. 2. For noncoordinated scheduling schemes, i.e. "SE-Or" and "GS-NC", the schedulers of each sector are not aware of other sectors. While for "SE-Or", BSs always transmit at full power, for "GSNC", BSs obtain their transmit powers for each sector without considering the possible co-channel interference they cause to impact other sectors. Therefore, for the non-coordinated schedulers, the transmit power increases with the number of small cells to combat interference. As for the coordinated scheduling schemes such as "SE-Co" and "GS-Co", the schedulers oversee the entire network to manage rather than combat interference.
In this case, the transmit power of one sector will be adjusted according to the power from others. When more small cells are present in the network, more interference occurs, and the transmit power is automatically reduced accordingly to meet the EE or SE performance.

\section{CONCLUSION}

The survey has shown that efforts have been made to address the EE problem in both single-tier macro network and twotier HetNet. With the presented results, we conclude that coordination between the BSs can achieve better EE. Apart from green scheduling in single cell scenario, there have been recent works focusing on coordinated multicell scenario [2], [5], [8]. The main challenge in the multicell scenario is the complexity while dealing with the co-channel interference, such that finding an optimal green scheduling solution is likely to be very challenging, if not impossible. In the HetNet scenario, most of the green scheduling investigations still focus solely on the small-cell tier; as such cross-tier coordination represents a worthwhile track of research. Our exploration of green scheduling has unveiled several interesting directions to be further researched:

(i) Developing a green scheduler with coordinated macro and small BSs.

(ii) Addressing the unfairness problem among different tiers.

(iii) Exploring other user selection/grouping scheme apart from greedy user selection, such as graph-based approach.

(iv) Reducing the complexity of scheduling algorithm. This is an important issue especially for densely deployed networks.

\section{REFERENCES}

[1] G. Fettweis and E. Zimmermann, "ICT energy consumption-trends and challenges", in Proc. 11th Int. Symp. on WPMC, 2008.

[2] F. Héliot, M. Imran, and R. Tafazolli, "Low-Complexity Energy-Efficient Resource Allocation for the Downlink of Cellular Systems", IEEE Trans. Commun., vol. 61, no. 6, pp. 2271-2281, 2013.

[3] A. Ambrosy, G. Auer, O. Blume, M. Carettiand et al., "D2. 2: definition and parameterization of reference systems and scenarios", INFSOICT247733 EARTH (Energy Aware Radio and NeTwork TecHnologies) Tech. Rep., 2010.

[4] D. Feng, C. Jiang, G. Lim, J. Cimini, G. Feng and G. Li, "A survey of energy-efficient wireless communications", IEEE Commun. Surveys Tuts., vol. 15, no. 1, pp. 167-178, 2013.

[5] L. Venturino, A. Zappone, C. Risi, and S. Buzzi, "Energy-Efficient Scheduling and Power Allocation in Downlink OFDMA Networks With Base Station Coordination", IEEE Trans. Wireless Commun., vol. 14, no. 1, pp. 1-14, 2015.

[6] J. Jiang, M. Peng, K. Zhang, and L. Li, "Energy-efficient resource allocation in heterogeneous network with cross-tier interference constraint", in Proc. IEEE 24th Int. Symp. on PIMRC Workshops, 2013.

[7] Z. Zhang, H. Zhang, Z. Zhao, H. Liu, X. Wen, and W. Jing, "Low complexity energy-efficient resource allocation in down-link dense femtocell networks", in Proc. IEEE 24th Int. Symp. on PIMRC, 2013.

[8] D. Ng, E. Lo, and R. Schober, "Energy-Efficient Resource Allocation in Multi-Cell OFDMA Systems with Limited Backhaul Capacity", IEEE Trans. Wireless Commun., vol. 11, no. 10, pp. 3618-3631, 2012.

[9] X. Xiao, X. Tao, Y. Jia, and J. Lu, "An energy-efficient hybrid structure with resource allocation in OFDMA networks", in Proc. IEEE WCNC, 2011. 
[10] Z. Ren, S. Chen, B. Hu, and W. Ma, "Energy-Efficient Resource Allocation in Downlink OFDM Wireless Systems With Proportional Rate Constraints", IEEE Trans. Veh. Technol., vol. 63, no. 5, pp. 2139-2150, 2014.

[11] X. Xiao, X. Tao, and J. Lu, "QoS-Aware Energy-Efficient Radio Resource Scheduling in Multi-User OFDMA Systems", IEEE Commun. Lett., vol. 17, no. 1, pp. 75-78, 2013.

[12] Z. Zheng, L. Dan, S. Gong, and S. Li, "Energy-efficient resource allocation for downlink OFDMA systems", in Proc. IEEE ICC Workshops, 2013.

[13] C. Xiong, G. Y. Li, S. Zhang, Y. Chen, and S. Xu, "Energy-Efficient Resource Allocation in OFDMA Networks", IEEE Trans. Commun., vol. 60, no. 12, pp. 3767-3778, 2012.

[14] L. Venturino, N. Prasad, and X. Wang, "Coordinated Scheduling and Power Allocation in Downlink Multicell OFDMA Networks", IEEE Trans. Veh. Technol., vol. 58, no. 6, pp. 2835-2848, 2009.

[15] D. Gesbert, S. Hanly, H. Huang, S. Shamai, O. Simeone, and W. Yu, "Multi-cell MIMO cooperative networks: A new look at interference", IEEE J. Sel. Areas Commun., vol. 28, no. 9, pp. 1380-1408, 2010.

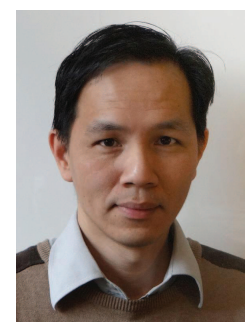

Chuan Heng Foh (S'00-M'03-SM'09) received his M.Sc. degree from Monash University, Australia in 1999 and Ph.D. degree from the University of Melbourne, Australia in 2002. After his PhD, he spent 6 months as a Lecturer at Monash University in Australia. In December 2002, he joined Nanyang Technological University, Singapore as an Assistant Professor until 2012. He is now a Senior Lecturer at the University of Surrey. His research interests include protocol design and performance analysis of various computer networks including wireless local area and mesh networks, mobile ad hoc and sensor networks, 5G networks, and data center networks. He has authored or coauthored over 100 refereed papers in international journals and conferences. He actively participates in IEEE conference and workshop organization. He is currently an Associate Editor for IEEE Access, IEEE Wireless Communications, and International Journal of Communications Systems. He is the Vice-Chair (Europe/Africa) of IEEE Technical Committee on Green Communications and Computing (TCGCC). $\mathrm{He}$ also chairs the Special Interest Group on Green Data Center and Cloud Computing under TCGCC. He is a senior member of IEEE.

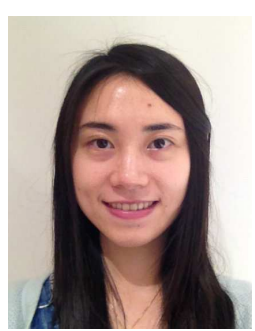

Ting Yang received her M.Sc degree in Mobile Communications Systems from University of Surrey, United Kingdom in 2013. She is currently working towards the Ph.D. degree in the Institute for Communication Systems (ICS), University of Surrey. Her research interests concern energy efficiency, user scheduling and radio resource management, especially for the densely deployed small cells.

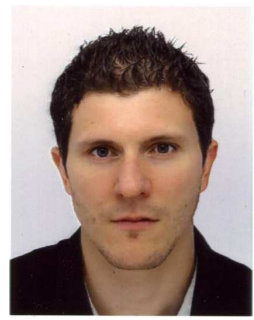

Fabien Héliot (S05-M07) received the Ph.D. degree in Mobile Telecommunications from Kings College London, in 2006. He is currently a Lecturer at the Institute for Communication Systems (ICS) of the University of Surrey. He has been actively involved in European Commission funded projects such as FIREWORKS, ROCKET, SMART-Net, EARTH and LEXNET. His main research interests are EMF exposure, energy efficiency, cooperative communication, MIMO, and radio resource management. 